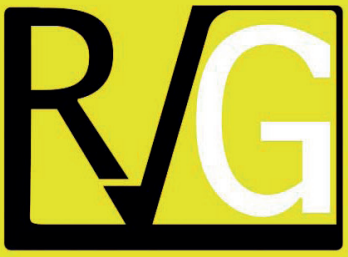

Julio - Septiembre, 2021

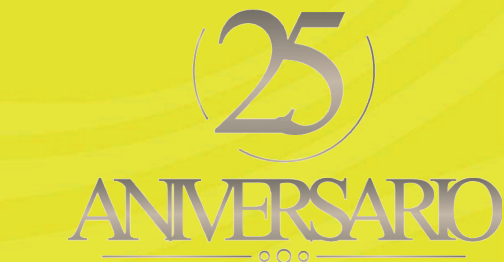

ANMERSARO
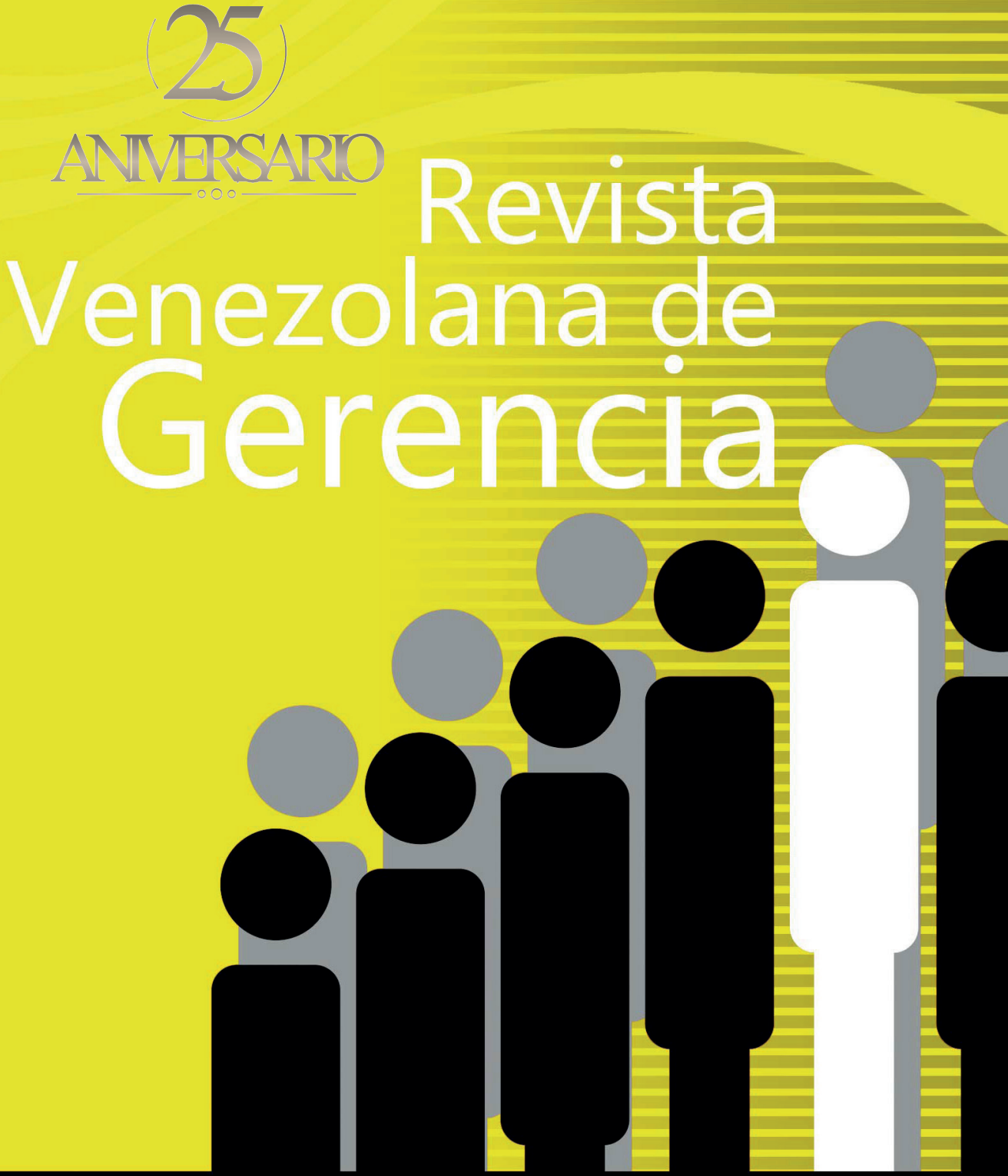

UNIVERSIDAD DEL ZULIA (LUZ)

Facultad de Ciencias Económicas y Sociales Centro de Estudios de la Empresa

ISSN 1315-99

Esta obra está bajo una licencia de Creative Comm Reconocimiento-NoComercial-Compartirlgual 3.0 Unpo http://creativecommons.org/licenses/by-nc-sa/3.0/deed.es 
COMO CITAR: Villa Castaño, L. E., Perdomo-Ortiz, J., y Pedraza, C. E. (2021). Responsabilidad social empresarial en empresas de la industria colombiana: una aproximación comprensiva. Revista Venezolana de Gerencia, 26(95), 918-942. https://doi.org/10.52080/

\author{
Universidad del Zulia (LUZ) \\ Revista Venezolana de Gerencia (RVG) \\ Año 26 No. 95 Julio-Septiembre 2021, 918-942 \\ ISSN 1315-9984 / e-ISSN 2477-9423
}

\title{
Responsabilidad social empresarial en empresas de la industria colombiana: una aproximación comprensiva
}

\author{
Villa Castaño, Lida Esperanza* \\ Perdomo-Ortiz, Jesús** \\ Pedraza, Cristian Enrique***
}

\section{Resumen}

La presente investigación tiene como objetivo interpretar los antecedentes y consecuentes de la responsabilidad social empresarial en empresas del sector industrial de Colombia. La investigación se realiza a través de una metodología cualitativa utilizando la encuesta semiestructurada a profundidad. Los datos se analizan en el software en NVivo utilizando cuatro categorías de análisis. Los resultados aportan evidencia al campo emergente de la responsabilidad social en países en desarrollo. Se encuentra que la responsabilidad social empresarial es antecedida por un sentido de deber moral y filantrópico centrado en valores de los empresarios fundadores, por la necesidad de adquirir licencias sociales para operar en territorios en conflicto, por la necesidad de fortalecer las relaciones con los principales grupos de interés primarios y por la necesidad de llenar vacíos institucionales. La responsabilidad social empresarial genera consecuentes en el diseño de modelos de negocio hacia formas hibridas y de creación de valor compartido, amplía los niveles de relacionamiento con grupos de interés

Recibido: 10.02 .21

Aceptado: 15.05 .21

* El presente artículo es resultado del proyecto de investigación titulado Causas y consecuencias de la responsabilidad social empresarial en Colombia.

** Doctora y magister en filosofía, economista, licenciada en filosofía y letras. Profesora investigadora, programa de administración de empresas, Universidad Cooperativa de Colombia, sede Bogotá. E-mail: lida.villacas@ campusucc.edu.co. Autor de correspondencia. ORCID: https://orcid.org/0000-0003-3510-3870

*** Doctor en economía y dirección de empresas empresa, administrador de empresas. Profesor titular, Departamento de administración de empresas, Pontificia Universidad Javeriana. E-mail: jesush.perdomo@ javeriana.edu.co ORCID: https://orcid.org/0000-0002-9947-5350

**** Magister en Gerencia de la responsabilidad social y sostenibilidad empresarial, filósofo. Profesor de cátedra, Pontificia Universidad Javeriana, Universidad Cooperativa de Colombia. E-mail: c.pedraza@javeriana.edu. co ORCID: https://orcid.org/0000-0002-1779-2863 
secundarios como las instituciones locales, las ONGs, o el entorno medio ambiental en crisis, y modifica los modelos de gobernanza corporativa al involucrar fehacientemente el proceso de rendición de cuentas cumpliendo estándares internacionales.

Palabras clave: Responsabilidad social empresarial; antecedentes; consecuentes; Colombia; industria

\title{
Corporate social responsibility in companies in Colombian industry: a comprehensive approach
}

\begin{abstract}
The present research aims to interpret the antecedents and consequences of corporate social responsibility in companies in the industrial sector of Colombia. The research is carried out through a qualitative methodology using an in-depth semistructured survey. The data is analyzed in the software at NVivo using four categories of analysis. The results of corporate social responsibility and provides evidence to the emerging field of corporate social responsibility in developing countries. It has been found that corporate social responsibility is preceded by a sense of moral obligation and philanthropy centered on the company's founding members' values, the necessity to acquire a social license to operate in a conflict zone, the necessity to strengthen relationships with primary stakeholders, and the necessity to fill institutional gaps. CSR has an impact on the design of business models for hybrid forms and the creation of shared value, it broadens relationships with secondary stakeholders, such as local institutions, NGOs, or the environmental context in crisis, and it modifies the models of corporate governance by reliably involving the process of accountability, abided by international standards.
\end{abstract}

Keywords: corporate social responsibility; backgrounds; consequences Colombia; industry

\section{Introducción}

La responsabilidad social empresarial (RSE) se ha consolidado como un campo de estudio muy activo en la disciplina del management (Aguinis y Glavas, 2012). El trabajo empírico es abundante y de todo tipo, y las preguntas de investigación son recurrentes sobre los efectos que tiene la implementación de prácticas de responsabilidad social sobre el desempeño organizacional (de Jong y van der Meer, 2017). La tradición ha privilegiado los efectos sobre el desempeño financiero (Wang et al. 2016), pero hoy día es claro que 
fue válida la pregunta fundamental de los años 90 sobre el desempeño social corporativo (Wood, 1991).

La aparición de la teoría de los grupos de interés (stakeholders) en los años 80 (Freeman, 1984) generó un interés por el lado de la demanda: los antecedentes de la RSE (Ali et al. 2017). Por ejemplo, surgió una importante línea de investigación alrededor de los intereses de los consumidores y cómo un consumidor más consciente obligaba a las empresas a pensar en la necesaria implementación de prácticas de RSE (Roberts, 1996; Villa et al. 2016). Lo mismo aconteció con las demandas por un desarrollo sostenible de proveedores o la necesidad de prácticas de gestión humana responsable (Ağan, 2016; Aguinis y Glavas, 2019). Recientemente la pregunta por la necesidad de la RSE se ha asociado a los cambios estratégicos de las empresas y la búsqueda de un nuevo valor compartido (Porter y Kramer, 2006).

Este estudio se interesa por comprender e interpretar los antecedentes y consecuentes de la RSE en un país emergente como Colombia donde el conflicto ha jugado un papel crucial en el desarrollo empresarial del país. Por tanto, los matices del origen de la RSE como sus impactos son particulares al contexto país. Además, busca aportar evidencia empírica en un campo de estudio emergente, el de la RSE en países en desarrollo (Ali et al. 2017; Jamali et al. 2017; Jamali y Karam, 2018; Aguinis et al. 2020). La delimitación de este nuevo campo de estudio se ha generado fundamentalmente en los estudios críticos del management (Banerjee, 2007; Banerjee, 2014), en la creciente literatura de empresa y paz (Oetzel et al. 2009; Kolk y Lenfant, 2010), y se ha fundamentado igualmente en la riqueza de los estudios transculturales (Cross-Cultural) (Singh y Del Bosque, 2008).

El valor agregado de la investigación se genera precisamente por las condiciones excepcionales de un contexto como el colombiano, donde la expresión de la RSE es contingente y matizada altamente por el entorno. Un entorno de los negocios formal y dinámico, pero constituido dentro de un ambiente institucional o sociopolítico complejo. En otras palabras, seguramente la expresión empresarial de la RSE en Colombia se ajusta a los parámetros identificados por la corriente principal (mainstream) de la literatura sobre RSE, pero el contexto especifico y la contingencia al entorno turbulento de un país en desarrollo, permiten identificar matices diferenciadores de los antecedentes y consecuentes de la RSE.

\section{Antecedentes y consecuentes de la RSE}

Dentro de la disciplina del management es importante pensar que el estudio de las prácticas empresariales son contingentes al contexto (Aguinis et al. 2020). De allí el auge de los estudios culturales comparados y de la vertiente paralela al mainstrem de los estudios críticos en management (Singh y Del Bosque, 2008; Banerjee, 2014). Para las líneas de investigación en RSE, entender que las prácticas empresariales definidas por la responsabilidad social podrían darse de manera explícita o implícita fue importante (Matten y Moon, 2008).

Por su parte, Ormaza et al. (2020) señala que se orienta a generar mejoras en la calidad de vida, salud y educación; en el ámbito económico, pretendiendo un crecimiento equitativo a través de la 
preservación de los ecosistemas en los cuales opera y de los cuales se beneficia, sin dejar de mencionar la responsabilidad que asumen las organizaciones en su relación directa que la relaciona intrínsecamente dependiente de la sociedad y su progreso (Rincón et al. 2018).

Una gerencia de la RSE explicita se pliega a lo que se denomina el "Caso de Negocio", mientras una gerencia de la RSE implícita se despliega en una importante simbiosis con el entorno institucional de los negocios. En otras palabras, para el estudio de la RSE el ambiente sociopolítico y cultural importa; más aún cuando desde sus inicios conceptuales y prácticos la RSE se entendió como una necesaria respuesta a la demanda de hechos sociales.

La evolución del concepto de la RSE (Mejía-Bermúdez y BomCamargo, 2019) va haciendo evidente la necesidad de pensar contextualmente la práctica empresarial. La concepción del empresario filántropo, o la pirámide de responsabilidades empresariales, o la gestión de grupos de interés, o la necesaria integración entre estrategia y sociedad, o la concepción de una ciudadanía corporativa, o la reciente apuesta por la sostenibilidad corporativa, evidencian la compleja, contextualizada y localizada práctica de la responsabilidad social. En todas las acepciones conceptuales de la RSE impera la respuesta empresarial a hechos sociales generados en los entornos inmediatos (Perdomo y Escobar, 2011; Latapí, Jóhannsdóttir \& Davídsdóttir, 2019).

En consecuencia, los estudios del management en el campo de la RSE encontraron que era relevante $y$ pertinente pensar y definir un campo de estudio específico de la RSE en el contexto de los países en desarrollo
(Lindgreen y Córdoba, 2010; Jamali y Karam, 2016; Alí et al. 2017; Jamali y Karam, 2018; Aguinis et al. 2020). Las razones son evidentes. Los países en desarrollo, como Colombia, son espacios territoriales altamente complejos y específicos en sus entornos locales. Allí las urgencias de investigación y prácticas empresariales están mediadas por la necesidad de inclusión social, reducción de la pobreza, creación de valor más allá del financiero, actividad empresarial en entornos de conflicto, violencia y corrupción; lo que se resume en la necesidad de una formulación estratégica en entornos para los negocios frágiles y complejos.

Esta complejidad del entorno es aún más difícil de comprender cuando se pone sobre la mesa la dualidad en términos de actividad económica; en los países en desarrollo, como es el caso de Colombia, coexiste un mundo urbano con un mundo rural, o de forma alterna, un mundo de formalidad económica paralelo a otro de informalidad y mera supervivencia (Visser, 2008; Dobers y Halme, 2009).

El estudio de la RSE en países en desarrollo como Colombia implica la reflexión sobre los siguientes puntos. Primero, el nivel de respuesta a las demandas sociales está altamente motivado por la ética de los negocios y la filantropía. Incluso en los acercamientos conceptuales y prácticos de la RSE como un Caso de Negocio, la identificación de hechos sociales para ligarlos a la estrategia, esta presionada por las altas demandas sociales y necesidades de poblaciones vulnerables que no están cercanas al core del negocio. Segundo, las expresiones gerenciales de la RSE adquieren formas hibridas; es decir, ya no es claro o evidente que la RSE sea solamente fruto de una apuesta en un 
Caso de Negocio, también responde a las presiones e interacciones en un entorno institucional pro-bienestar social sin el fin de lucro.

Las prácticas de RSE son una hibridación entre aquellas pensadas y ejecutadas desde dentro del negocio como respuesta específica a las demandas de grupos de interés, y aquellas que cumplen un rol de ejecución de los contratos sociales locales o nacionales, en los que la empresa es un actor relevante. Tercero, la puesta en práctica de la RSE ya no solo busca resultados en términos de eficiencia operativa o financiera, sino que las dimensiones del resultado empresarial se deben leer igualmente en función de la búsqueda de legitimidad de la empresa con una licencia social para operar. Cuarto, la gestión de los grupos de interés está altamente matizada por los denominados grupos de interés secundarios.

Es decir, aunque la gestión de grupos de interés hace una identificación de la cadena de valor e identifica los requerimientos de los grupos de interés primarios, aquellos que tienen una relación funcional con el core del negocio, en los países en desarrollo, como Colombia la gestión de grupos de interés se ve altamente permeada por los intereses de grupos de interés secundarios o también denominados de influencia o de regulación. Aquí la comunidad, los medios, las ONGs, el medioambiente, o el gobierno local y nacional son actores principales en la construcción de la relación negociosociedad. Quinto, la RSE en los países en desarrollo llena vacíos institucionales; o de otra manera, la empresa asume roles de agente de desarrollo local.

El Estado es débil y en este sentido otros actores clave como el sector privado, e incluso actores violentos, deben llenar vacíos de servicios sociales como la educación, la salud, la justicia, o las mismas capacidades de emprendimiento de las comunidades locales. En suma, en los países en desarrollo la RSE se expresa de manera particular, contingente, y especifica al contexto. En ellos los territorios están fracturados en lo social, y el nivel de desarrollo y la institucionalidad es muy disímil. Las grandes empresas formalizadas, aun estando en un entorno moderno y seguro, deben asumir los retos de cadenas de valor operando en territorios fracturados.

\subsection{Antecedentes de la RSE}

Existe abundante literatura de revisión sistemática y de metaanálisis sobre antecedentes y consecuentes de la RSE (Sprinkle y Mainess, 2010; Wang y Juslin, 2011; Aguinis y Glavas, 2012; Fifka, 2013; Jamali y Karam, 2016; Jain y Jamali, 2016). Para este ejercicio comprensivo, se consideran cuatro categorías para agrupar los antecedentes y consecuentes. (Ver cuadro 1). 


\section{Cuadro 1 \\ Categorías de Antecedentes y Consecuentes de la RSE}

\begin{tabular}{|c|c|c|}
\hline Categorías & Antecedentes de la RSE & Consecuentes de la RSE \\
\hline \multirow{3}{*}{ Estrategia } & Ajuste al modelo de negocio & Desempeño social corporativo \\
\hline & Búsqueda de Reputación / Imagen & Rentabilidad / Reputación \\
\hline & Ajuste a la Razón de ser & Creación de valor compartido \\
\hline \multirow{3}{*}{ Relacionamiento } & Respuesta a demandas sociales & $\begin{array}{l}\text { Gestión y diálogo con grupos de in- } \\
\text { terés }\end{array}$ \\
\hline & Construcción de relaciones & Construcción de confianza \\
\hline & Desarrollo de grupos de interés & Licencia social \\
\hline \multirow{3}{*}{ Responsabilidad } & Atención a la regulación pública & Cumplimiento de estándares \\
\hline & Búsqueda de trasparencia & Rendición de cuentas \\
\hline & Apuesta por la filantropía & $\begin{array}{l}\text { Fortalecimiento del sentido de deber } \\
\text { moral }\end{array}$ \\
\hline \multirow{3}{*}{ Gobierno y Gestión } & Necesidad de un nuevo modelo de gestión & Cambio en el modelo de gestión \\
\hline & Necesidad de ajuste al gobierno corporativo & Cambio en el gobierno corporativo \\
\hline & $\begin{array}{l}\text { Necesidad de ajuste a la estructura orga- } \\
\text { nizacional }\end{array}$ & Cambio en la estructura organizacional \\
\hline
\end{tabular}

Fuente: Elaboración propia

Con respecto a los antecedentes, el primer grupo se refieren a los cambios en la estrategia de la organización. Cuando la empresa interioriza los compromisos sociales, también se obliga a la implementación de prácticas de responsabilidad social (Bansal y Roth, 2000; Lindgreen y Córdoba, 2010; Porter y Kramer, 2011). En este mismo sentido, la modificación de la razón de ser (misión, visión, valores) motiva la inclusión de prácticas socialmente responsables (Rishi y Moghe, 2013; LinHi y Muller, 2013).

Esta circunstancia engloba a la RSE como una oportunidad de negocio.
Igualmente, dentro de la estrategia de la RSE, surgen motivadores como la necesidad de apostar por la mejora de la imagen reputacional de la empresa, el incremento del valor de marca, o la visibilidad pública del negocio (Du et al. 2016). Este conjunto de antecedentes se ve matizado en la implementación de la RSE en países en desarrollo, al considerar un entorno de los negocios con grandes fallas de mercado, y la desigual construcción de mercados por la impronta de desequilibrios económicos y sociales en estos.

El segundo grupo se concentra en la categoría del relacionamiento 
estratégico. La implementación de prácticas de RSE en las organizaciones requiere previamente de la construcción de relaciones de confianza con los diferentes grupos de interés. De allí que expresiones alternas de la RSE, p.ej. con consumidores o proveedores, deriven de la construcción de relaciones en la cadena de valor y estas a su vez motivan la consolidación de sistemas específicos de responsabilidad social (Rupp et al. 2006; Baden et al. 2009). En el caso de los países en desarrollo, como Colombia, las empresas por supuesto que construyen relacionamiento estratégico en las cadenas de valor con sus grupos de interés primarios, pero además requieren de un ejercicio de construcción de licencias sociales en comunidades o territorios en conflicto con altas desigualdades (Gutiérrez y Jones, 2004).

Un tercer grupo de antecedentes de la RSE se asocia al clásico modelo de las responsabilidades (Carroll, 1979); englobadas en la categoría de la responsabilidad. La RSE se promueve en las organizaciones debido a la necesidad de responder con suficiencia a las obligadas responsabilidades económicas y legales. En este sentido la regulación económica y legislativa obliga a la implementación de prácticas de RSE. Se obliga a las empresas a promover la perdurabilidad del negocio y la generación de empleo, así como se obliga a la transparencia y rendición de cuentas.

\section{Bajo el mismo motivante} de responsabilidades obligadas y voluntarias, las empresas encuentran deseable el seguimiento a un orden moral, filantrópico y con sentido de justicia social. Aquí la expresión de los principios y valores de la ética de los negocios obliga la implementación de modelos de RSE (Jamali et al. 2017; Carroll y Brown, 2018). En territorios en conflicto, complejos y con altos niveles de desigualdad, las responsabilidades se expresan con mayor fuerza y obligación al considerar el valor del altruismo y la necesaria construcción de comunidad.

El cuarto grupo de antecedentes de la RSE se deriva de las formas de gobiernos corporativos y las nuevas apuestas de modelos de gestión (categoría de Gobierno y Gestión). El surgimiento de la teoría de los grupos de interés obligó a repensar las formas de gobernanza empresarial. Los problemas de agencia se multiplicaron y complejizaron, dando lugar a cambios en los estamentos de gobierno al considerar los intereses adicionales de los grupos de interés (Acosta y Pérezts, 2019; Jo y Harjoto, 2011). De estos cambios se deriva el surgimiento de liderazgos responsables, de nuevos compromisos organizacionales $y$, en general, de una apuesta por una nueva cultura organizacional ligada a estándares de RSE: nacionales e internacionales (Jamali y Sidani, 2008).

En el caso de países en desarrollo, como Colombia, la gobernanza y los modelos de gestión para la RSE deben incorporar el sentido de bienestar social obligado por los entornos y contextos de desigualdad.

\subsection{Consecuentes de la RSE}

Del lado de los consecuentes de la RSE, la literatura muestra un marco similar al de los antecedentes (Chang, 2015; Arya y Mittendorf, 2015; Adámek, 2016; Islam et al. 2016; Asante et al. 2019; Humala y Humala, 2020). El primer grupo se deriva de los cambios en la estrategia. Aunque el campo de la responsabilidad social 
parece inmerso en imperativos morales, como la transparencia, la honestidad y el altruismo, la implementación de la RSE se convierte en una forma de Caso de Negocio. La implementación de prácticas de responsabilidad social promueve oportunidades de negocio alineadas al bienestar social, las cuales pueden rentabilizarse en los mercados. En este sentido, y a raíz de los cambios en la forma de competir y de generar valor, la literatura evidencia que las empresas, por ejemplo, han desarrollado programas de mercadeo con causa y/o de mercadeo social. El mercadeo con causa se halla representado por acciones orientadas a resolver un problema social, desde el mismo modelo; el mercadeo social separa la acción social del core del negocio. Por esto, es acertado afirmar que la relación entre empresa y sociedad ha cambiado; las empresas han decidido apartarse de las posturas asistencialistas, y la responsabilidad social se concibe como una creación de valor compartido o como una sostenibilidad organizacional donde las cuestiones sociales se encuentran alineadas a la rentabilidad económica (Porter y Kramer, 2002; Porter y Kramer, 2011; Montiel y Delgado-Ceballos, 2014).

Este primer grupo de consecuentes englobados en la categoría de la estrategia además incluye los cambios en la imagen y reputación derivados de la RSE. La literatura registra que la implementación de la RSE es una forma de mejorar la reputación de la empresa frente a las audiencias de interés (Belt y Paolillo, 1982; Stanaland et al. 2011). En países en desarrollo, con un entorno de los negocios alterado por conflictos y desigualdades sociales, los consecuentes englobados en la categoría de la estrategia obligan a pensar en desempeños corporativos más allá de lo financiero, y a crear modelos de negocios híbridos expresados en creación de valor compartido.

El segundo grupo de consecuentes se categoriza en el relacionamiento con los grupos de interés. La implementación de prácticas de RSE se convierte en un buen predictor de la mejora de la relación de confianza y diálogo con los grupos de interés (Fassin, 2009; Rupp et al. 2006). El ejercicio de la responsabilidad social implica establecer canales de comunicación entre empresa y sociedad (comunidades locales) para identificar necesidades y alinearlas con los objetivos de la organización; todo con el fin de merecer una licencia social (Gutiérrez y Jones, 2004; Miles et al. 2006). Adicionalmente, el enfoque de negocios en la RSE conlleva una apuesta por la construcción de confianza en la cadena de suministro (desarrollo sostenible de proveedores), por la implementación de buenas prácticas medioambientales con foco en la economía circular, y por el respeto de los derechos humanos como la estrategia base para mejorar la relación con los trabajadores con compromiso organizacional (Pierce y Madden, 2005; Carmeli et al. 2007; Seuring, 2013; Jamali et al. 2015)

Además, la implementación de la RSE, si atiende las expectativas de los consumidores, mejorara la relación con base en un consumo responsable (Maignan et al. 1999; Carmeli et al. 2007). Los consecuentes derivados por el relacionamiento con los grupos de interés en los países en desarrollo se deben contextualizar por la existencia de vacíos institucionales, y por la mayor visibilidad de grupos de interés secundarios como agentes locales, ONGs, medios de comunicación, o el mismo entorno medioambiental en 
deterioro.

El tercer grupo de consecuentes de la RSE se engloba en la categoría de las responsabilidades. Luego de consolidar un modelo de responsabilidad social, el empresario transita por los eslabones de la pirámide de Carroll (1979), y consecuente con el cumplimiento de las responsabilidades económicas, legales, morales y filantrópicas (sociales), las empresas promueven un proceso de rendición de cuentas, una visibilización de su deber moral expresado en una ciudadanía corporativa, y en ultimas adoptan estándares que facilitan todo el proceso de accountability (Jamali y Carroll, 2017; Carroll y Brown, 2018; Villa y Herrera, 2020). En los países en desarrollo los consecuentes de la RSE vistos desde la categoría de la responsabilidad, acentúan el sentido de la responsabilidad filantrópica y de los modelos de negocio híbridos; el desequilibrio social tan pronunciado lleva a las empresas a dedicar recursos explícitos de inversión social sin expectativas de rentabilidad en el corto plazo.

Finalmente, el cuarto grupo de consecuentes se engloban en la categoría de gobierno y gestión. Introducir prácticas de RSE en las operaciones de las empresas lleva progresivamente a modificaciones en los modelos de gestión y en la estructura organizacional (departamentos de sostenibilidad). Incluso se ha propuesto que los modelos enfocados en la responsabilidad social pueden asimilarse a modelos de excelencia empresarial (Waddock y Graves, 1997). Y cuando la RSE se involucra en el direccionamiento estratégico de las empresas, ésta afecta todo el proceso de toma de decisiones $\mathrm{y}$ de gobierno corporativo (Aguinis y Glavas, 2012; Acosta y Pérezts, 2019).
Interpretar los consecuentes de la RSE en el gobierno y gestión de las empresas de países en desarrollo, implica enfrentarse a estructuras y modelos de negocio híbridos, donde la creación de valor se da en el trípode la economía, lo social y lo ambiental, pero además donde la filantropía juega un papel importante.

\section{Consideraciones metodológicas}

Este artículo asume un enfoque de investigación cualitativo con un diseño de carácter comprensivointerpretativo. El ejercicio exploratorio partió de la definición de las categorías de antecedentes y consecuentes de la RSE, para después alimentarlas con los datos cualitativos del trabajo de campo realizado. El procesamiento de la información se realizó en Nvivo y a través de la interpretación de los autores se realizó triangulación de datos evidenciando las categorías de análisis: antecedentes y consecuentes de la RSE.

La fuente de datos fue la aplicación de un instrumento de entrevista semiestructurado a profundidad, y el contenido de cada categoría se fue dando en función de un ejercicio de comparación permanente a partir de la lectura individual de cada uno de los investigadores. En este sentido, se buscó identificar el rico detalle del dato de la entrevista individual y al mismo tiempo capturar los posibles patrones emergentes.

La aplicación del instrumento de entrevista estuvo precedida de un ejercicio de documentación de cada una de las empresas y, en particular, de la lectura detallada de los reportes de sostenibilidad (RSE). Las entrevistas tuvieron un tiempo promedio deaplicación de 75 minutos y se estructuraron en dos 
partes. Una inicial de generalidades de la RSE en cada una de las empresas, para luego indagar por los eventos que antecedieron a la puesta en práctica de la RSE, y las consecuencias de ésta.

La muestra teórica se constituyó por ocho empresas con sede en Bogotá (Colombia) que debían cumplir con tres criterios de inclusión: i) que fuera una empresa altamente formalizada y de tamaño grande; ii) que fuera una empresa que hubiera realizado por lo menos tres reportes de sostenibilidad; y iii) que perteneciera al sector industrial con importante experiencia de operaciones en el país. Las razones de estos criterios de inclusión implicaban una muestra de empresas homogéneas donde el negocio y el modelo de gestión estaban consolidados; las empresas evidenciaban una apuesta explícita por la RSE y sus prácticas; y todas configuraban una cadena de valor larga y con múltiples stakeholders y relaciones.

El perfil del informante fue una persona del nivel directivo del área de sostenibilidad (RSE) y asuntos corporativos con más de cinco años de antigüedad en el cargo. En la Tabla 2 se caracteriza la muestra de las empresas del trabajo de campo evidenciando el tipo de empresa, los años de operación en Colombia y el año del primer informe de sostenibilidad. El último criterio revela la formalización de la RSE.

\section{Tabla 2}

Muestra teórica de empresas del estudio

\begin{tabular}{|c|c|c|c|c|}
\hline ID & Subsector & Tipo & $\begin{array}{c}\text { Años de } \\
\text { operación en } \\
\text { Colombia }\end{array}$ & $\begin{array}{c}\text { Primer } \\
\text { Informe de } \\
\text { Sostenibilidad }^{1}\end{array}$ \\
\hline E1 & Alimentos y bebidas & $\begin{array}{l}\text { Multinacional } \\
\text { colombiana }\end{array}$ & 75 & 2009 \\
\hline E2 & Alimentos y bebidas & $\begin{array}{l}\text { Multinacional } \\
\text { extranjera }\end{array}$ & 131 & 2010 \\
\hline E3 & $\begin{array}{l}\text { Limpieza y cuidado } \\
\text { personal }\end{array}$ & $\begin{array}{l}\text { Multinacional } \\
\text { extranjera }\end{array}$ & 33 & 2012 \\
\hline E4 & $\begin{array}{l}\text { Insumos para la } \\
\text { construcción }\end{array}$ & $\begin{array}{l}\text { Multinacional } \\
\text { extranjera }\end{array}$ & 24 & 2012 \\
\hline E5 & $\begin{array}{c}\text { Cerámica e insumos para } \\
\text { el hogar }\end{array}$ & $\begin{array}{l}\text { Multinacional } \\
\text { colombiana }\end{array}$ & 139 & 2011 \\
\hline E6 & $\begin{array}{l}\text { Insumos para la } \\
\text { construcción }\end{array}$ & $\begin{array}{l}\text { Multinacional } \\
\text { extranjera }\end{array}$ & 58 & 2014 \\
\hline E7 & Alimentos y bebidas & $\begin{array}{l}\text { Multinacional } \\
\text { colombiana }\end{array}$ & 100 & 2011 \\
\hline E8 & $\begin{array}{l}\text { Insumos para la } \\
\text { construcción }\end{array}$ & $\begin{array}{l}\text { Multinacional } \\
\text { colombiana }\end{array}$ & 86 & 2009 \\
\hline
\end{tabular}

1 Datos tomados de databasegri donde se evidencia el año del primer informe y todos los reportes de las empresas que siguen esta metodología y cuelgan en la página los informes de manera anual

Fuente: elaboración propia 


\section{RSE en empresas del sector industrial en Colombia: Hallazgos, antecedentes y consecuentes}

El trabajo empírico mostró que en empresas grandes del sector industrial en Colombia se han formalizado los procesos de gestión de la RSE, lo anterior al hacer visible esta gestión a través de los reportes de sostenibilidad bajo la metodología GRI. También se encontró relevante la larga trayectoria empresarial, al evidenciarse la importancia de los fundadores en la concepción filantrópica de la RSE en las empresas de capital colombiano; incluso en la actual gerencia formal, de las multinacionales nacionales e internacionales, se mantienen los rasgos del deber moral en el comportamiento responsable de las empresas. En la presentación de los resultados y la discusión de los antecedentes y consecuentes de la RSE en empresas industriales, confirmará la relevancia de una gestión y diálogo con los grupos de interesa más allá de los involucrados en la cadena de valor, y la nueva concepción de formas hibridas en los modelos de negocio.

\subsection{Antecedentes de la RSE en Colombia}

Los antecedentes de la RSE en empresas del sector industrial colombiano evidencian que

desde el punto de vista estratégico los factores intrínsecos que hacen parte de la razón ser de la organización o extrínsecos como la estrategia y el valor compartido, son los elementos que impulsaron inicialmente la RSE.

"Las causas de la RSE en la empresa...

si yo hablo de una organización de hace

setenta años, pues desde que arrancó, porque la principal responsabilidad social estaba en dar unos buenos productos, en dar un producto que no fuera inocuo, no estar contaminando a la gente, no estar mal nutriendo a la gente. Sí, el principal aporte social de una empresa tiene que estar en su razón de ser". (E1, Alimentos y bebidas).

"Hacer responsabilidad social debe nacer de una gestión transparente, de una gestión ética, de unos valores corporativos, de un valor por el empleado, de un valor por las comunidades donde está activa la organización". (E5, Cerámica).

Al asociar la RSE a la razón de ser de la organización las empresas E1 y E5 expresan que hay una sintonía natural en lo que es la organización y la causa de ser socialmente responsable. En este sentido, por un lado, la consideración de que los productos de calidad son la base ontológica de la RSE y, de otro lado, la cultura organizacional soportada por los valores corporativos, reflejan que la causa del surgimiento de la RSE es intrínseca al negocio y no está asociada a modas, presiones del mercado o problemas del contexto particular de la organización. La RSE es el ADN de la organización.

Ahora bien, dentro de la evidencia encontrada juega un papel relevante las causas estratégicas asociadas a la necesidad de alinear la RSE al core del negocio y la búsqueda de una generación de valor compartido.

"Creemos que la filantropía no genera unos retornos de capital social, resultados. La filantropía no construye redes, no construye capital social, no mejora la situación. Nosotros hacemos programas de alto impacto que generan retorno en el futuro. Por ejemplo, una mejora en las áreas". (E1, Alimentos y bebidas).

"La causa por la cual nosotros introducimos este tema es la 
construcción de consciencia de la empresa de generar valor a los grupos de interés y a la empresa". (E7, Alimentos y bebidas).

La percepción de los directivos refleja los siguientes aspectos: i) una fuerte separación entre filantropía y la oportunidad de mejorar el negocio concebido en relación con la amplitud del retorno de capital social y la construcción de redes. ii) La consciencia de que la empresa no se limita a la generación de riqueza para un solo actor. Se expande, entonces, la comprensión del negocio integrando el conocimiento de que en el desarrollo del negocio se crea valor para la empresa y los grupos de interés y, iii) La rentabilidad no es el motor de inicio de la RSE, es la generación de valor que implica superar la visión de no dañar a la sociedad para crecer de manera conjunta.

El relacionamiento con la comunidad, los empleados y proveedores jugaron un papel determinante para el nacimiento de la RSE.

"En Colombia y en la región un pilar grande y muy importante para nosotros se llama desarrollo comunitario. Ese pilar tiene un objetivo muy claro: asegurar o mantener la continuidad operativa desde el componente social, la mayoría del presupuesto de inversión social está destinada a ese eje estratégico. Las comunidades no necesitan regalos, necesitan oportunidades". (E4, Construcción)

"La atención de las necesidades de la comunidad es la causa más importante de la inclusión de la RSE en la empresa. Llega un punto en el que nuestro cliente externo se vuelve casi igual de importante al interno debido a que por diferentes maneras se ve afectado por las plantas de producción". (E2, Alimentos y bebidas).

"Considero que la más importante del inicio de la RSE en nuestra compañía es la atención de las necesidades de los empleados dado a que somos todos un recurso fundamental de la empresa $y$, por lo tanto, debemos cuidar el personal en temas de clima organizacional, respeto, compromiso y sobre todo en cuanto al bienestar". (E3, Limpieza y cuidado personal).

"La RSE empieza formalmente hace setenta años en la relación con nuestro proveedor principal. No digo que todo se haya hecho perfecto, pero seguramente se han hecho cosas muy bien en la dinámica desde los sesentas, setentas, ochentas. Capacitaciones, visitas, asesorías sobre cómo manejar los insumos, cómo debe ser el proceso de recolección y almacenaje de materia prima perecedera. Cómo vender la materia prima y ser más competitivos". (E1, Alimentos y bebidas).

El relacionamiento con las comunidades está ligado al objetivo de obtener la licencia social, así la empresa puede operar sin que la comunidad interfiera en el desarrollo del negocio. La comunidad (vecinos, grupos comunitarios locales) es impactada directamente por las actividades de la empresa, no es un actor segundario incide de modo directo en el desarrollo de la operación; la comunidad se convierte en un actor primario en tanto de ella depende, de algún modo, la licencia para operar. Por tanto, la empresa la percibe como un stakeholder con poder legítimo que se convierte en una de las causas por las cuales emerge la RSE. En particular, la forma de relacionamiento con este grupo de interés no se cimienta en una relación filantrópica al estilo de una donación, se hace inversión social que está conectada con el desarrollo de capacidades o de negocios inclusivos en la base de la pirámide y tiene como propósito mejorar las condiciones de vida de una población marginada social y económicamente. Así, entonces, la relación con la comunidad se convierte en un eje estratégico del negocio 
necesario para construir confianza y simultáneamente operar.

Otro hallazgo importante es la consideración de que los empleados son la parte vital para el desarrollo de la RSE. En este sentido, la base del cuidado del empleado como la apuesta por el bienestar y el clima organizacional configuran lo que se denomina la justicia organizacional. De otro lado, el relacionamiento con los proveedores va más allá de la relación comercial ya que se enfoca en eldesarrollo de capacidades técnicas que es estratégico para la compañía en tanto la materia prima que se compra sigue los estándares de calidad requeridos. Se subraya del hallazgo en el relacionamiento con el proveedor, que la empresa hace una apuesta por fortalecer este grupo de interés como una estratégica para asegurar la calidad del producto, pero también como una transferencia de conocimiento que le permite al proveedor ser más competitivo.

En la categoría de la responsabilidad como antecedente de la responsabilidad los directivos entrevistados afirman:

De otro lado, en el ámbito explícito de la responsabilidad los directivos entrevistados afirman:

"Esta es una empresa familiar y desde la filosofía y los valores que tienen los dueños, en su estructura administrativa y estratégica, el tema de apoyo social y filantropía fue una base importante para la organización; históricamente el tema de responsabilidad social ha sido uno de los temas prioritarios para los accionistas de la organización". (E5, Cerámica).

"Somos una empresa de ciento ochenta años en el mercado, treinta de ellos en Colombia y nuestra iniciativa y estrategia de RSE viene desde que se creó la empresa. A nivel de Colombia tenemos una fundación que trabaja en temas de educación y de emprendimiento en lugares marginales del país". (E3, Limpieza y cuidado personal).

La filantropía como causa de inicio de la RSE no está directamente relacionada con el ámbito del negocio. Son acciones que contribuyen con el mejoramiento de un problema de la sociedad pero que no está relacionado con el core del negocio. En este sentido, se resalta la vocación del empresario filántropo que ayuda per se a la razón de ser de la empresa. Sin embargo, el segundo hallazgo refleja que la apuesta por la creación de una fundación es paralela a la vocación de la empresa en términos de su sentido social.

Desde el punto de vista del seguimiento a la ley se marcan dos escenarios:

"La empresa siempre ha tenido especial interés por los temas ambientales con un enfoque de cumplimiento de ley". (E2, Alimentos y bebidas).

"Iniciamos el ciclo de responsabilidad social a fondo a partir del año 2009 dado a que la compañía adquirió el compromiso con la sostenibilidad y nos permitió adherirnos al pacto global, alinearnos con los 10 principios universalmente aceptados". (E6, Construcción)

La regulación medioambiental y el Pacto Global constituyen dos fuentes de emergencia de la RSE. Bajo el primer enfoque, la empresa se sitúa en el ámbito legal y el cumplimiento de la normatividad constituye el arranque de ser socialmente responsables. De otro lado, la adherencia voluntaria a Pacto Global implica un compromiso con ser sostenible en el mercado respetando los derechos humanos en su conjunto. De alguna manera los principios de Pacto Global jalonan a la empresa a empezar el camino hacia la RSE. 
El gobierno corporativo como órgano encargado de dar directrices relacionados con el norte y la toma de decisiones, también, ha jugado un papel central a la hora de incorporar la RSE:

"Iniciamos prácticas de RSE

básicamente porque cambiamos de dueño, los dueños llegaron y dijeron: esto es lo que hay que hacer de ahora en adelante y, eso es lo que estamos haciendo. Con la llegada y compra por parte de la multinacional de nuestra empresa en el año 2005 se impuso la estrategia de sostenibilidad de la casa matriz, y esa fue la que adoptamos como guía y es la que ha desarrollado hasta la fecha". (E2, Alimentos y bebidas).

La directriz del gobierno corporativo refleja no solo una apuesta por la alineación de la subsidiaria a la estrategia de la casa de matriz sino una evidente declaración por incluir una visión de sostenibilidad propia de la compañía. Aquí el antecedente no viene del contexto donde opera la organización, sino que proviene del más alto órgano directivo.

De otro lado, y con respecto al modelo de gestión la creación de una nueva área dentro de la empresa marca la formalización de una visión ampliada de la RSE.

"En la empresa se empieza a pensar en un área que se dedique únicamente a los temas de RSE y que gerencie estos temas. En el año 2010 se crean el área de sostenibilidad corporativa para la organización". (E5, Cerámica).

Estructurar la RSE se da a partir de un cambio en el modelo de gestión a tal punto de crear una nueva área dentro de la organización. Por tanto, la RSE se incorpora a la operación de la empresa como una necesidad de ajustarse a las demandas del nuevo diseño organizacional.

Al revisar las causas por las cuáles se integra la RSE a las empresas vemos que ellas discurren entre lo estratégico, lo relacional, la responsabilidad, el gobierno corporativo y el modelo de gestión. Se encuentra que la RSE es antecedida por un sentido de deber moral y filantrópico centrado en valores de los empresarios fundadores, por la necesidad de adquirir licencias sociales para operar en territorios en conflicto, por la necesidad de fortalecer las relaciones con los principales grupos de interés primarios, y por la necesidad de llenar vacíos institucionales.

\subsection{Consecuentes de la RSE en Colombia}

Los consecuentes de la implementación de la RSE en las empresas analizados desde la categoría de la estrategia se orientaron hacia: i) el cambio en la estrategia (posicionamiento), ii) la mejora de la reputación e imagen y, iii) la mejora de la rentabilidad y la ventaja competitiva. En términos de cambio en la estrategia, esto fue lo que afirmaron los entrevistados:

"Hoy que cambiamos nuestra misión y nuestra esencia incluimos la RSE y eso dice mucho de una empresa". (E1, Alimentos y bebidas)

"La RSE Ha cambiado en torno a que antes era como más filantropía, hoy día estamos enfocado hacia una estrategia de negocio y con unas metas claras, indicadores claros y enfocados en proyectos y en programas que tengan impacto y no disgregado en varios proyectos que no tengan el mejor impacto en la sociedad". (E3, Limpieza y cuidado personal)

"La consecuencia de la haber implementado la RSE es que la estrategia cambia y se enfoca en dos temas que teníamos rezagados: los temas sociales y ambientales. Estos cobran gran fuerza en la estrategia de la organización y logran empezar 
a desarrollar cambios Importantes de gestión y de forma de gestionar procesos a nivel interno". (E5, Cerámica).

La integración de la RSE en la empresa ha implicado grandes cambios. Por un lado, el ajuste de la misión orientada a un compromiso por la RSE deja entrever un cambio cualitativo de lo que es el negocio. De otro lado, un desplazamiento de la filantropía para considerar que la RSE es una aliada de la estrategia y, por tanto, es susceptible de ser medida. En concreto, la materialización de la RSE se hace evidente en la medición de los impactos y en la integración de las esferas sociales y medioambientales.

Ahora bien, la solidez de la implementación de la RSE trae consigo una mayor reputación que es otorgada por terceros a través de distinciones y premios o por en el reconocimiento de los millennials, generación que ha incluido en sus criterios de selección de empresas atractivas para trabajar a aquellas que son socialmente responsables. Estos supuestos se hacen explícitos a través de las percepciones de los directivos:

"Tenemos la participación en premios y casos de estudio relacionados con temas de sostenibilidad $y$, en los últimos tres años, nos hemos ganado doce premios relacionados con responsabilidad social y sostenibilidad; y estamos posicionarnos como líderes de sostenibilidad en Colombia y en la región". (E4, Construcción)

"En nuestra compañía los empleados arrancan desde la universidad, son los famosos millennials. Ellos son los que más exigen en el tema de responsabilidad social, entonces que nosotros tengamos una estrategia de RSE nos ha beneficiado en la medida en que estos estudiantes quieran ingresar a una compañía socialmente responsable. Nuestra compañía ha atraído porque la gente nos valora y ha mejorado nuestra reputación en torno a que somos socialmente responsables". (E7, Alimentos y bebidas).

"La empresa ganó en el año 2014 el reconocimiento PREAD, Programa de Excelencia Ambiental Distrital en la categoría Élite, por su desempeño e iniciativas ecoeficientes. EI PREAD es liderado por la Secretaría Distrital de Ambiente y la Alcaldía Mayor de Bogotá. La relación con los grupos de interés ha traído cambios significativos totalmente positivos con los cuales hemos venido ganando premios, prestigio y mucho reconocimiento a nivel mundial". (E6, Construcción).

En cuanto al consecuente de la RSE en la rentabilidad y la ventaja competitiva estas son las relaciones encontradas:

"Si soy una empresa responsable y en el mismo sector encuentro que hay empresas que no lo son obviamente mejora mi rentabilidad y la ventaja competitiva frente a otras empresas". (E2, Alimentos y bebidas).

"Hoy tenemos camiones con menos emisiones y que consumen menos gasolina, queremos hacer ventas de una manera sostenible, estamos llevando productos de un portafolio amplio que permite tener productos para todos los gustos, con azúcar, sin azúcar, permitimos esa variedad". (E1, Alimentos y bebidas).

Las consecuencias del ejercicio de la RSE se reflejan en la rentabilidad y la ventaja competitiva, este atributo pone a la empresa en un mejor posicionamiento en el mercado. De otro lado, la inclusión de un transporte más amigable con el medioambiente reduce los costos de operación de la logística de distribución $\mathrm{y}$, por tanto, mejora el rendimiento financiero. Finalmente, la ampliación de la gama de productos más saludables, sin excluir los tradicionales, le permite a la empresa ampliar su ventaja competitiva como el segmento del mercado. 
La RSE tiene como consecuente una ampliación de la visión del negocio que conlleva la integración de los stakeholders dentro de la estrategia corporativa. De allí que las siguientes afirmaciones den cuenta de los diálogos con los grupos de interés y de su importancia para la empresa.

"Hemos tenido diferentes

conversaciones con los principales grupos de interés; hemos revisado las expectativas de ellos versus la relevancia de sus temas para nosotros, encontrando un punto de encuentro en la matriz de materialidad". (E8, Construcción).

"En nuestra relación con los stakeholder manejamos unos temas de conflicto, pero lo hacemos de una manera muy ética, muy madura. Buscamos una comunicación fluida con los líderes para que ganen ellos y nosotros. Hemos hecho resolución de conflictos exitoso que nos ha permitido que ellos sigan teniendo confianza en las cosas que la compañía hace". (E3, Limpieza y cuidado personal).

El relacionamiento con los stakeholders se mueve en varios ámbitos. En un primer lugar, se evidencia que a través de conversaciones la organización desvela las expectativas de los grupos de interés para posteriormente cruzar las demandas de estos con la matriz de materialidad. Se infiere que estos diálogos confirman lo que la empresa ya ha detectado como una posible demanda de los diferentes actores que impacta. En segundo lugar, la relación con los stakeholders implica una búsqueda de acuerdos donde ambas partes ganan y en tercer lugar, conocer las necesidades y construir comunidad de apoyo mutuo y participativo. En general, este relacionamiento muestra no solo la apertura de laorganización para conocer e integrar los stakeholders sino una relación de carácter colaborativo.
Con respecto a la responsabilidad social interna, que tiene que ver con el bienestar de los empleados, son varios los consecuentes que ha traído la implementación de la RSE:

"Lo más importante son los programas que se generan para los empleados y para su bienestar pues son parte importantísima de la estrategia". (E4, Construcción).

"El mantener unas relaciones laborales fundamentales en la colaboración $y$ el trato digno, reconocido por los trabajadores, básicamente en la comunicación por el respeto, el cuidado de su seguridad, su constante capacitación y el aporte a la educación de sus hijos, ha sido interpretado por los colaboradores del norte del Cauca como un reconocimiento de su dignidad". (E6, Construcción).

"Los colaboradores ya saben a nivel de grupo ¿Qué es lo que estamos haciendo? ¿Cómo trabajamos? Entonces aumentan las consultas, es más fácil comunicarse, tenemos una base sólida real de datos con la cual conversar". (E7, Alimentos y bebidas).

De otro lado, las comunidades se han convertido en uno de los actores más álgidos en el desarrollo de la operación del negocio. Por tanto, el relacionamiento de la empresa con este grupo de interés ha traído para las empresas analizadas un nuevo horizonte de comprensión y de relacionamiento.

"La comunidad nos da los lineamientos de lo que hay que hacer en las comunidades, a través de los diálogos que tenemos con ellos cada dos años. $Y$ nosotros los invitamos a diferentes espacios para conocer su percepción. A raíz de esos diálogos concluimos que cuando vamos a hacer un proyecto en territorio las comunidades son las que deben apropiarse de esos proyectos, y si se necesita mano de obra los primeros que vamos a contratar son a ellos". (E1, Alimentos y bebidas).

"Entender que la sostenibilidad trasciende un kit escolar, trasciende 
una donación que se le puede hacer a una comunidad, pone a la empresa realmente a pensar cuáles son los impactos de mi negocio. Si yo estoy impactando el agua de mis comunidades cómo voy a hacer para gestionar eso responsablemente, cómo hago para disminuir el consumo, cómo hago para asegurarme que las comunidades donde yo esté también tengan acceso al agua". (E8, Construcción)

"La búsqueda de tener un mejor relacionamiento en el lugar donde vamos a llegar; es decir, unos buenos vecinos que llegan a sumar y a contribuir con el desarrollo local, que para nosotros es clave manejar esto, en tripartita con el estado. No generamos dependencia, pero si buscamos que se genere un desarrollo en estas comunidades por medio de los elementos más básicos del desarrollo junto con el estado, como es el transporte, la educación, la alimentación, la vivienda. Nosotros nos aseguramos de que las comunidades tengan un crecimiento". (E4, Construcción).

El diálogo es la vía para el acercamiento y conocimiento de los intereses de la comunidad. En este sentido, las empresas han propiciado espacios de encuentro en el que se integran los intereses de estos actores en el desarrollo de proyectos comunitarios. De otro lado, la forma de relación entre empresa y comunidad no está mediada por la donación sino por la comprensión de los impactos que genera la operación del negocio buscando mitigar los mismos. Finalmente, la relación empresa, comunidad y Estado es la forma más eficiente de generar redes de apoyo para mitigar problemas estructurales, de un país emergente, como la vivienda y la educación.

Enlacategoría delaresponsabilidad se identificó un consecuente de la RSE en términos del mercadeo con causa relacionado con el manejo del agua. No obstante, se subraya que la iniciativa proviene de uno de los sectores que más usa el mismo recurso.

"El manejo que tenemos con la fundación del cuidado del agua, al ser un tema tan predominante, a nivel no solamente país si no a nivel mundial, nos hace diferentes dentro del sector con respecto a las demás empresas. El hecho de que los niños desde pequeños tengan presente la importancia del cuidado del agua nos hace ser mucho más sostenibles en el largo y mediano plazo". (E6, Construcción).

Educar en el cuidado del agua a los adultos del mañana puede ayudar a transformar la forma de relacionarse con el entorno natural. La apuesta de la empresa se interpreta como la acción desprovista de interés en tanto no está relacionada con el core del negocio; así, entonces, el consecuente implica una consciencia macro que exige un esfuerzo de parte de todos los actores por ayudar a proteger los recursos naturales.

Se encontró evidencia de consecuentes sobre la gobernanza y el modelo de gestión.

"Al llegar los temas de sostenibilidad a la estrategia empezamos a focalizarnos en tres temas relevantes: lo económico, lo social y lo ambiental. Antes hacíamos los reportes económicos que tiene toda compañía, pero no hacíamos algunos informes o no compartíamos información acerca de los otros dos temas que, cuando llega la sostenibilidad, se vuelven más relevantes para la organización". (E5, Cerámica)

"Hemos encontrado oportunidades de mejora en eficiencia, hemos encontrado oportunidades de conocer mejores prácticas porque vemos que tal vez otra compañía lo hace de una manera más interesante que podríamos evaluar, esto nos ha abierto la posibilidad de compararnos, también el hecho de que podamos retarnos. No solo estar comparándonos con 
compañías de Colombia sino con compañías en otros países que hacen cosas muy distintas. Entonces uno de los mayores beneficios es que estamos midiendo el riesgo. No quiere decir que lo eliminemos, pero sí nos permite generar estrategias con las cuales vayamos acercándonos a nuestras metas". (E8, Construcción)

La inclusión de la RSE impactó el modelo de negocio y gobierno en los siguientes aspectos: la integración de metas e indicadores de carácter social y medioambiental en la gestión de la empresa; el mejoramiento en eficiencia y la medición del riesgo del negocio permitiendo disminuir los impactos negativos derivadas de las actividades empresariales. En este último escenario el reconocimiento de los asuntos materiales, impactos más relevantes derivados de la operación del negocio, permite hacer una ruta de mejoramientos en la gestión de la organización.

La interpretación de los discursos de la RSE en Colombia relativos a los antecedentes y consecuentes confirma las particularidades de un territorio altamente fragmentado en lo económico, lo social y lo ambiental (Ali et al. 2017; Jamali et al. 2017; Jamali y Karam, 2018). Se hacen evidentes los discursos robustos de la RSE, asimilados a la creación de valor compartido y la sostenibilidad, cuando se argumenta desde el foco de los mercados urbanos y formales (Porter y Kramer, 2011). Sin embargo, las amplias cadenas de valor de las empresas también muestran que aguas bajo y aguas arriba, la expresión de la RSE está más ligada a aquella que caracteriza a empresas localizadas en países en desarrollo.

En particular, la comunidad como un grupo de interés que en la corriente principal de la RSE es un actor secundario, en Colombia es claramente visible y se convierte en un actor primario (Fassin, 2009; Ali et al. 2017). Una lectura de la RSE desde allí lleva a destacar: i) que mucho del grado de respuesta a las demandas sociales esta permeada por la filantropía; ii) que la expresión gerencial de la RSE toma matices híbridos entre el Caso de Negocio y la necesaria imbricación con lo institucional y lo contextual del territorio; iii) la necesidad de hacer explicita una licencia social legitima; y iv) a descubrir el papel de la empresa como un agente de desarrollo local con funciones paralelas en la oferta de servicios públicos.

En la categoría Estrategia los antecedentes de la RSE se asocian a la necesidad de hacer explícitos en la misión del negocio los valores familiares, de tradición o de responsabilidad ante las demandas sociales. La RSE se hace parte de la razón de ser del negocio. Aquí la RSE se expresa en la figura de empresarios filántropos o en la constitución de fundaciones paralelas al negocio. En este sentido, la RSE surge como una respuesta desde una ética de la filantropía, propia de los países en desarrollo (Gutiérrez y Jones, 2004; Lindgreen et al. 2010). A manera de contraste, una lectura desde el entorno moderno de las empresas lleva a entender que el antecedente estratégico de la RSE es el despliegue del principio de valor compartido. Seguramente, esta lectura del antecedente de la RSE está asociada al seguimiento de la rendición de cuentas a través de la metodología GRI. Sin embargo, aquí también entran a jugar matices de la RSE en países en desarrollo. A la creación de valor compartido se le antepone el principio de transparencia, de obligación moral y del no-daño a la comunidad (Lin-Hi y Müller, 2013).

Los consecuentes de la 
implementación de la RSE en la categoría de la estrategia se asocian a la inclusión de la RSE como sostenibilidad, y a la necesaria relación clásica de la RSE y el desempeño social corporativo (Wood, 2010). Aquí se expresa la RSE dentro del mainstream que aboga por las fuentes de ventajas competitivas, de reputación y de mayor valor en los mercados. Nuevamente, el matiz sobre los consecuentes estratégicos de la RSE lo da el sentido de comunidad en el territorio de operación del negocio, y la necesaria asociación del riesgo empresarial con el riesgo social y ambiental.

En la categoría Relacionamiento se destacan como antecedentes de la RSE la búsqueda de una licencia social, la legitimación de la operación, y la construcción de un entorno de bienestar social (Gutiérrez y Jones, 2004. Claramente, este discurso surge cuando se piensa en el despliegue de la cadena de valor, en particular aguas arriba. Allí se hace explicito que la gestión de los grupos de interés trasciende a los primarios y en consecuencia se construyen diálogos con la comunidad en un sentido de desarrollo de proveedores con fomento de capital social; surgen como interlocutores válidos los líderes sociales del territorio. En los consecuentes se hace explicito la mejora en la comunicación y construcción de confianza tanto con grupos de interés primarios $y$ secundarios. Además, y de una manera notoria, se plantea el surgimiento de una responsabilidad social interna centrada en los trabajadores. Allí destaca la mejora el sentido del trato digno y la extensión del bienestar del trabajador a su entorno familiar y comunitario (Jamali et al. 2015).

EnlacategoríaResponsabilidad, los antecedentes de la RSE se pliegan a las clásicas responsabilidades económicas (generar utilidades y empleo) legales, morales y discrecionales. Por lo discutido arriba, la jerarquía de responsabilidad se asocia al deber moral de no hacer daño y cumplir con los preceptos de los valores familiares o de tradición. Esto es particularmente claro en las empresas con origen y propiedad colombiano (Gutiérrez y Jones, 2004). Mientras los consecuentes de la responsabilidad se asocian fundamentalmente al premio o reconocimiento por seguir algún estándar de la RSE; por ser responsable con el seguimiento a la ley o al mercado. El matiz en esta categoría de análisis es que lo ambiental, entendido como un grupo de interés secundario, llena gran parte del orgullo responsable de las empresas. $Y$ nuevamente, el territorio de operación con su especificidad de sociedad lleva a la búsqueda de reconocimiento de los gobiernos locales (Gutiérrez y Jones, 2004).

Finalmente, en la categoría de gobierno y gestión los antecedentes se pliegan a la necesidad de formalización de las áreas de RSE; y a la inclusión de nuevas formas de gobernanza dadas por la involucración de las demandas de grupos de interés a la toma de decisiones, o por la fusión, adquisición o directrices de las matrices internacionales (Acosta y Pérezts, 2019). Aquí el sentido de la RSE desde el mainstream es evidente. El único matiz a la gestión y gobernanza es la demanda en voz alta de las comunidades en los territorios de provisión y/o extracción de las materias primas. Los consecuentes se asocian a gobiernos corporativos más permeados por los entornos institucionales del país, por las condiciones del contexto social y político, y por la creciente legitimidad de las comunidades como un actor principal 
allende de las cabeceras urbanas donde se asientan las matrices.

\section{Conclusiones}

Este trabajo aporta a la discusión de la RSE en países en desarrollo. Se encontró evidencia de una expresión diferencial de la RSE en Colombia tomando una muestra homogénea de empresas de sector industrial. En particular, la RSE en Colombia plantea una respuesta a las demandas sociales altamente permeada por criterios del deber moral, filantropía y ética empresarial. Asimismo, se expresa dentro de un modelo de negocio hibrido con rasgos de Caso Empresarial y resultados pro-bienestar social, siempre con la intención de legitimar su accionar en los territorios donde se expanden las cadenas de valor a través de búsquedas de licencias sociales. La RSE en Colombia se expresa en un diálogo con grupos de interés más allá de los constitutivos de la cadena de valor; y como era de esperar por lo dicho en la literatura, las empresas se convierten bajo circunstancias particulares, y proyectos, en agentes de desarrollo local.

Este trabajo tiene limitaciones y retos asociados al tipo de estudio. Un estudio cualitativo invita a la validación con otras muestras teóricas de empresas en Colombia que hagan explicita su apuesta por la RSE; y donde el sector y el tamaño pueden incluir otras variantes de análisis. El sesgo de interpretación y análisis propio de estos estudios llevan a plantear trabajos comparados en países en desarrollo, como una forma agregada de triangulación. Y la limitación de la representatividad de los resultados es una clara invitación a la contrastación empírica de corte cuantitativo.

\section{Referencias}

Acosta, P., y Pérezts, M. (2019). Unearthing sedimentation dynamics in political CSR: The case of Colombia. Journal of Business Ethics, 155(2), 425-444. https:// link.springer.com/article/10.1007/ s10551-017-3502-x

Adámek, P. (2016). Consequences of Corporate Social Responsibility Approach in Voluntary European and Global "Responsible" Initiatives. In Proceedings of the 3rd International Conference on European Integration (pp. 19-27).

Ağan, Y., Kuzey, C., Acar, M. F., y Açıkgöz, A. (2016). The relationships between corporate social responsibility, environmental supplier development, and firm performance. Journal of Cleaner Production, 112, 1872-1881. https://doi.org/10.1016/i. jclepro.2014.08.090

Aguinis, H., Villamor, I., Lazzarini, S. G., Vassolo, R. S., Amorós, J. E., y Allen, D. G. (2020). Conducting Management Research in Latin America: Why and What's in It for You?. SAGE Journals. Collection. https://doi.org/10.25384/ SAGE.c. 4842345

Aguinis, H., y Glavas, A. (2012). What we know and don't know about corporate social responsibility: A review and research agenda. Journal of management, 38(4), 932-968. https:// doi.org/10.1177/0149206311436079

Aguinis, H., y Glavas, A. (2019). On corporate social responsibility, sensemaking, and the search for meaningfulness through work. Journal of Management, 45(3), 1057-1086. https://doi. org/10.1177/0149206317691575

Ali, W., Frynas, J. G., y Mahmood, Z. (2017). Determinants of corporate 
Villa Castaño, Lida Esperanza; Perdomo-Ortiz, Jesús; Pedraza, Cristian Enrique Responsabilidad social empresarial en empresas de la industria colombiana: una aproximación comprensiva

social responsibility (CSR) disclosure in developed and developing countries: A literature review. Corporate Social Responsibility and Environmental Management, 24(4), 273-294. https://doi.org/10.1002/ csr. 1410

Arya, A., y Mittendorf, B. (2015). Supply chain consequences of subsidies for corporate social responsibility. Production and Operations Management, 24(8), 1346-1357. https://doi.org/10.1111/poms.12326

Asante, E., He, Z., Boadi, E. K., Bosompem, J., y Avornyo, P. (2019). Consequences of corporate social responsibility on employees: The moderating role of work motivation patterns. Personnel Review, 49(1), 231-249. https://www.emerald.com/ insight/content/doi/10.1108/PR-082018-0288/full/html

Baden, D. A., Harwood, I. A., y Woodward, D. G. (2009). The effect of buyer pressure on suppliers in SMEs to demonstrate CSR practices: an added incentive or counter productive? European Management Journal, 27(6), 429-441. https://doi. org/10.1016/j.emj.2008.10.004

Banerjee, S.B. (2007). Corporate social responsibility: the good, the bad and the ugly. Critical Sociology, 34(1), 51-79. https://doi. org/10.1177/0896920507084623

Banerjee, S.B. (2014). A critical perspective on corporate social responsibility: Towards a global governance framework. Critical Perspectives on International Business, 10(1/2), 84-95. https:// www.emerald.com/insight/content/ doi/10.1108/cpoib-06-2013-0021/full/ $\underline{\mathrm{html}}$

Bansal, P., y Roth, K. (2000). Why companies go green: A model of ecological responsiveness. Academy of management journal, 43(4), 717736. https://doi.org/10.2307/1556363

Belt, J. A., y Paolillo, J. (1982). The influence of corporate image and specificity of candidate qualifications on response to recruitment advertisement. Journal of Management, 8, 105-112. https://doi. org/10.1177/014920638200800107

Carmeli, A., Gilat, G., y Waldman, D. A. (2007). The role of perceived organizational performance in organizational identification, adjustment and job performance. JournalofManagementStudies, 44(6), 972-992. $\quad$ https://doi.org/10.1111/ j.1467-6486.2007.00691.x

Carroll, A. B. (1979). A threedimensional conceptual model of corporate performance. Academy of management review, 4(4), 497-505. https://www.jstor.org/stable/257850

Carroll, A. B., y Brown, J. A. (2018). Corporate social responsibility: A review of current concepts, research, and issues. Corporate Social Responsibility: Business and Society 360, (2), 39-69. https://doi.org/10.1108/S2514175920180000002002

Chang, C.-H. (2015). Proactive and reactive corporate social responsibility: antecedent and consequence. Management Decision, 53 (2), 451-468. https://doi. org/10.1108/MD-02-2014-0060

de Jong, M. D., y van der Meer, M. (2017). How does it fit? Exploring the congruence between organizations and their corporate social responsibility, CSR) activities. Journal of business ethics, 143(1), 71-83. https://link.springer.com/ article/10.1007/s10551-015-2782-2

Dobers, P., y Halme, M. (2009). Corporate social responsibility and developing 
countries. Corporate Social Responsibility and Environmental Management, 16(5), 237-249. https:// doi.org/10.1002/csr.212

Du, X., Chang, Y., Zeng, Q., Du, Y., y Pei, H. (2016). Corporate environmental responsibility, CER) weakness, media coverage, and corporate philanthropy: Evidence from China. Asia Pacific Journal of Management, 33(2), 551-581. https://ideas.repec.org/a/kap/asiapa/ v33y2016i2d10.1007 s10490-0159449-5.html

Fassin, Y. (2009). The stakeholder model refined. Journal of Business Ethics, 84(1), 113-135. https:// link.springer.com/article/10.1007/ s10551-008-9677-4

Fifka, M. S. (2013). Corporate responsibility reporting and its determinants in comparative perspective-a review of the empirical literature and a meta $\square$ analysis. Business strategy and the environment, 22(1), 1-35. https://doi. org/10.1002/bse.729

Freeman, R. E. (1984). Stakeholder management: a strategic approach. New York: Pitman.

Gutiérrez, R., y Jones, A. (2004). Corporate social responsibility in Latin America: An overview of its characteristics and effects on local communities. In Contreras, M.E, Corporate social responsibility in the promotion of social development: Experiences from Asia and Latin America, Washington, Inter-American Development Bank, 151-187.

Humala Rojas, H. y Humala Rengel, J. (2020). La RSC responsabilidad social corporativa: factores específicos en la reputación de la empresa cuencana. Revista de Comunicación de la SEECl, 51, 109-128. https://doi.org/10.15198/

\section{seeci.2020.51.109-128}

Islam, T., Ahmed, I., Ali, G., y Sadiq, T. (2016). Behavioral and psychological consequences of corporate social responsibility: need of the time. Social Responsibility Journal, 12(2). https://www.emerald.com/insight/ content/doi/10.1108/SRJ-04-2015$\underline{0053 / f u l l / h t m l}$

Jain, T., y Jamali, D. (2016). Looking inside the black box: The effect of corporate governance on corporate social responsibility. Corporate governance: an international review, 24(3), 253-273. https://doi. org/10.1111/corg.12154

Jamali, D. R., EI Dirani, A. M., y Harwood, I. A. (2015). Exploring human resource management roles in corporate social responsibility: The CSR-HRM co-creation model. Business Ethics: A European Review, 24(2), 125-143. https://doi.org/10.1111/beer.12085

Jamali, D., Karam, C., Yin, J., y Soundararajan, V. (2017). CSR logics in developing countries: Translation, adaptation and stalled development. Journal of World Business, 52(3), 343-359. https://doi.org/10.1016/j. jwb.2017.02.001

Jamali, D., y Carroll, A. (2017). Capturing advances in CSR: Developed versus developing country perspectives. Business Ethics: A European Review, 26(4), 321-325. https://doi. org/10.1111/beer.12157

Jamali, D., y Karam, C. (2016). CSR in developed versus developing countries: a comparative glimpse. In Research Handbook on Corporate Social Responsibility in Context. Edward Elgar Publishing.

Jamali, D., y Karam, C. (2018). Corporate social responsibility in developing countries as an emerging field of study. International Journal of 
Villa Castaño, Lida Esperanza; Perdomo-Ortiz, Jesús; Pedraza, Cristian Enrique Responsabilidad social empresarial en empresas de la industria colombiana: una aproximación comprensiva

Management Reviews, 20(1), 32-61. https://doi.org/10.1111/ijmr.12112

Jamali, D., y Sidani, Y. (2008). Classical vs. modern managerial CSR perspectives: insights from Lebanese context and cross. cultural implications. Business and Society Review, 113(3), 329-346. https://doi.org/10.1111/j.14678594.2008.00323.x

Jo, H., y Harjoto, M. A. (2011). Corporate governance and firm value: The impact of corporate social responsibility. Journal of business ethics, 103(3), 351-383. https:// link.springer.com/article/10.1007/ s10551-011-0869-y

Kolk, A., y Lenfant, F. (2010). MNC reporting on CSR and conflict in Central Africa. Journal of Business Ethics, 93(2), 241- 255. https:// link.springer.com/article/10.1007/ s10551-009-0271-1

Latapí Agudelo, M.A., Jóhannsdóttir, L. \& Davídsdóttir, B. A (2019). Literature review of the history and evolution of corporate social responsibility. Int $J$ Corporate Soc Responsibility, 4(1). https://doi.org/10.1186/s40991-0180039-y

Lindgreen, A., y Córdoba, J. R. (2010). Corporate social responsibility in Latin America. Journal of business ethics, 167-170. https://publications.iadb. org/publications/english/document/ Corporate-Social-Responsibilityin-Latin-America-ResponsibleSolutions-to-Business-and-SocialProblems.pdf

Lin-Hi, N., y Müller, K. (2013). The CSR bottom line: Preventing corporate social irresponsibility. Journal of Business Research, 66(10), 19281936. https://www.sciencedirect. com/science/article/abs/pii/ $\underline{\mathrm{S} 0148296313000398}$
Maignan, I., Ferrell, O. C., y Hult, G. T. M. (1999). Corporate citizenship: Cultural antecedents and business benefits. Journal of the Academy of Marketing Science, 27(4), 455-469. https://link.springer.com/article/10.1 177/0092070399274005\#additionalinformation

Matten, D., and Moon, J. (2008) "Implicit" and "Explicit" CSR: A Conceptual Framework for a Comparative Understanding of Corporate Social Responsibility. Academy of Management Review, 33(2). https:// doi.org/10.5465/amr.2008.31193458

Mejía-Bermúdez, A., y Bom-Camargo, Y. I. (2019). Responsabilidad Social Empresarial para la apertura de mercados internacionales. Revista Venezolana de Gerencia, 2, 251269. https://doi.org/10.37960/revista. v24i2.31492

Miles, M. P., Munilla, L. S., y Darroch, J. (2006). The role of strategic conversations with stakeholders in the formation of corporate social responsibility strategy. Journal of Business Ethics, 69(2), 195$205 . \quad$ https://link.springer.com/ article/10.1007/s10551-006-9085-6

Montiel, I., y Delgado-Ceballos, J. (2014). Defining and measuring corporate sustainability: Are we there yet? Organization y Environment, 27(2), 113-139. https://doi. org/10.1177/1086026614526413

Oetzel, J., Westermann-Behaylo, M., Koerber, C., Fort, T. L., y Rivera, J. (2009). Business and peace: Sketching the terrain. Journal of business ethics, 89(4), 351$373 . \quad$ https://link.springer.com/ article/10.1007/s10551-010-0411-7

Ormaza Andrade, J., Ochoa Crespo, J., Ramírez Valarezo, F., \& Quevedo Vázquez, J. (2020). Responsabilidad social empresarial en el Ecuador: 
Abordaje desde la Agenda 2030. Revista De Ciencias Sociales (Ve), 26(3), 175-193. https://doi. org/10.31876/rcs.v26i3.33241

Pierce, M., y Madden, K. (2005). Driving success: Human resources and sustainable development. World Business Council for Sustainable Development. https://www.cisl. cam.ac.uk/resources/archivepublications/human-resources-andsustainable-development

Porter, M. E., and Mark R. K. (2002). The Competitive Advantage of Corporate Philanthropy. Harvard Business Review, 80(12), 56-69. https:// www.hbs.edu/faculty/Pages/item. aspx?num $=46631$

Porter, M. y Kramer, M (2006). Strategy and Society. Harvard Business Review, 84(12), 78-92. https://www. compromisoempresarial.com/wpcontent/uploads/strategy-society.pdf

Porter, M. y Kramer, M.R. (2011). How created shared value. Havard Business Review, 2-17. https:// sharedvalue.org.au/wp-content/ uploads/2015/12/Harvard-BusinessReview-Creating-Shared-Value.pdf

Rincón Quintero, Y., Montoya Álvarez, D. \& Vélez Patiño, P. (2018). Estrategias de Responsabilidad Social Empresarial en organizaciones del sector construcción en Medellín. Retos Revista de Ciencias de la Administración y Economía, 8(16), 79-94. https://10.17163/ret. n16.2018.06

Rishi, P., y Moghe, S. (2013). Integrating corporate social responsibility and culture as a strategy for holistic corporate success in India. Journal of Corporate Citizenship, (51), 17-37. https://doi.org/10.9774/ GLEAF.4700.2013.se.00004

Roberts, J. A. (1996). Will the real socially responsible consumer please step forward?. Business horizons, 39(1), 79-84. https://doi.org/10.1016/ S0007-6813(96)90087-7

Rupp, D. E., Ganapathi, J., Aguilera, R. V., y Williams, C. A. (2006). Employee reactions to corporate social responsibility: An organizational justice framework. Journal of Organizational Behavior, 27(4), 537-543. https://www.jstor.org/ stable/4093915

Seuring, S. (2013). A review of modeling approaches for sustainable supply chain management. Decision Support Systems, 54, 1513-1520. https://doi. org/10.1016/i.dss.2012.05.053

Singh, J., y Del Bosque, I. R. (2008). Understanding corporate social responsibility and product perceptions in consumer markets: A cross-cultural evaluation. Journal of Business Ethics, 80(3), 597611. https://link.springer.com/ article/10.1007/s10551-007-9457-6

Sprinkle, G. B., y Mainess, L. A. (2010). The benefits and cost of corporate social responsibility. Business Horizons, 53(5), 445-453. https://doi. org/10.1016/j.bushor.2010.05.006

Stanaland, A. J., Lwin, M. O., y Murphy, P. E. (2011). Consumer perceptions of the antecedents and consequences of corporate social responsibility. Journal of business ethics, 102(1), 47-55. https://link.springer.com/ article/10.1007/s10551-011-0904-z

Villa Castaño, L. E., Perdomo-Ortiz, J., Duenas Ocampo, S., y Duran Leon, W. F. (2016). Socially responsible consumption: An application in Colombia. Business Ethics: A European Review, 25(4), 460-481. https://doi.org/10.1111/beer.12128

Villa Castaño, L. E., y Herrera Romero, W. R. (2020). Estándares 
Villa Castaño, Lida Esperanza; Perdomo-Ortiz, Jesús; Pedraza, Cristian Enrique Responsabilidad social empresarial en empresas de la industria colombiana: una aproximación comprensiva

internacionales de rendición de cuentas social: entre la justificación y el apresamiento directivo. Innovar, 30(75), 135-150. https://doi. org/10.15446/innovar.v30n75.83262

Waddock, S. A., y Graves, S. B. (1997). The corporate social performancefinancial performance link. Strategic management journal, 18(4), 303-319. https://www.jstor.org/stable/3088143

Wang, L., y Juslin, H. (2011). The effects of value on the perception of corporate social responsibility implementation: A study of Chinese youth. Corporate Social Responsibility and Environmental Management, 18(4), 246-262. https:// doi.org/10.1002/csr.250
Wang, Q., Dou, J., y Jia, S. (2016). A metaanalytic review of corporate social responsibility and corporate financial performance: The moderating effect of contextual factors. Business $y$ Society, 55(8), 1083-1121. https:// doi.org/10.1177/0007650315584317

Wood, D. J. (1991). Corporate social performance revisited. Academy of management review, 16(4), 691-718. https://doi.org/10.2307/258977

Wood, D. J. (2010). Measuring corporate social performance: A review. International journal of management reviews, 12(1), 50-84.

https://onlinelibrary. wiley.com/doi/10.1111/j.14682370.2009.00274.x 